\title{
Melting transitions in isotropically confined three-dimensional small Coulomb clusters
}

\author{
S. W. S. Apolinario* and F. M. Peeters ${ }^{\dagger}$ \\ Departement Fysica, Universiteit Antwerpen, Groenenborgerlaan 171, B-2020 Antwerpen, Belgium
}

(Received 22 June 2007; published 10 September 2007)

\begin{abstract}
Molecular dynamic simulations are performed to investigate the melting process of small three-dimensional clusters (i.e., systems with one and two shells) of classical charged particles trapped in an isotropic parabolic potential. The confined particles interact through a repulsive potential. We find that the ground-state configurations for systems with $N=6,12,13$, and 38 particles interacting through a Coulomb potential are magic clusters. Such magic clusters have an octahedral or icosahedral symmetry and are found to have a large stability against intrashell diffusion leading to an intershell melting transition prior to the intrashell and radial melting process. For systems with two shells a local radial melting of subshells is found at low temperatures resulting in a structural transition leading to an increased symmetry of the ordered system. Using Lindemann's criterion the different melting temperatures are determined and the influence of the screening of the interparticle interaction was investigated. A normal mode analysis is performed and some of the normal modes are found to be determinantal for the melting process.
\end{abstract}

DOI: 10.1103/PhysRevE.76.031107

PACS number(s): 64.60.-i, 63.22.+m

\section{INTRODUCTION}

Wigner crystallization was predicted in 1934 and states that a liquid to solid phase transition should occur in a threedimensional electron gas at low temperature and density due to strong Coulomb repulsion [1]. This phase transition became known as Wigner crystallization and the solid phase as Wigner or Coulomb crystals. Wigner crystallization and properties of Coulomb crystals have been studied for decades in such a variety of systems as electron gas trapped on top of liquid helium [2], electrons trapped in quantum well structures [3], strongly coupled rf dusty plasmas [4], vortex clusters in an isotropic superfluid [5], laser-cooled trapped ion systems [6,7], dusty plasmas [8], etc. Formation of ordered clusters with nested shells is expected to occur in expanding neutral plasmas $[9,10]$.

Charged particles forming large three-dimensional (3D) isotropic clusters arrange themselves in two different forms. The center of the cloud is characterized by a body centered cubic lattice, while particles close to the border form concentric spherical shells, and on the shell's surface particles create a hexagonal lattice with few dislocations and disclinations. It has already been known for some time that finite systems have lower melting temperature than infinite systems [11]. Naturally, the question appears whether the lowering of the melting temperature is a consequence of the different form of ordering, or of the finite size. Recently, Ref. [12] has shown through a molecular dynamic simulation of larger clusters, that the decrease in melting temperature is proportional to the relative size of the shell surface. Additionally, the author showed that in the vicinity of the melting temperature, the diffusion rate in the outer regions is substantially larger parallel to the surface than perpendicular to it.

Concentric and equally spaced shells, which carry a specific number of particles, form small 3D systems. The num-

\footnotetext{
*sergio.apolinario@ua.ac.be

†rancois.peeters@ua.ac.be
}

ber of shells depends on the total number of particles and in general the number of shells increases with the number of particles. The ground state configuration of systems up to 12 particles consists of a single shell. These configurations in fact form three-dimensional regular polygons. From $N=13$ to 60 the arrangement of particles in the ground state configuration form two shells except for the clusters with $N=58$ and 59 particles. For systems larger than 60 particles groundstate configurations start to appear with three shells. For a review about the static properties of 3D clusters see Refs. $[13,14]$. The dynamics of small $3 \mathrm{D}$ clusters is expected to have different properties from that of large clusters due to finite size and symmetry effects which are stronger in small clusters. In the present paper we study, in detail, the melting process in 3D small Coulomb clusters.

A large number of works are directed toward 2D systems [15-17]. The melting process in 2D systems was found to happen through two distinct stages. First, at low temperature, particles of different shells lose their relative angular orientation, which is called intershell melting. Second, at higher temperature, particles are able to jump between shells, which is called radial melting. Reference [15] showed that some of the ground-state configurations in 2D systems, called magic clusters, exhibit a substantial larger intershell melting temperature. The stability found in magic clusters was due to the existence of commensurability between particles in different shells. The melting process in small 3D isotropic crystals have been much less explored. Reference [18] investigated only the melting process for clusters with $N=30$ and 94 particles. They found that for increasing temperature, the system shows two types of diffusion pattern: a first one is diffusion of particles inside the same shell and the second one concerns intershell diffusion. In our investigation we considered clusters having only one shell, i.e., clusters with $N=5$ to 12 particles and clusters with two shells and those having $N$ $=19,32,38$, and 55 particles were investigated in detail. Differently from previous works we investigated the melting process using the Lindemann's criterion for intrashell, intershell as well as radial displacements. Such analysis allowed us to identify a melting transition which was not found in 
Ref. [18], i.e., the intershell melting transition. Furthermore, we found that the melting process at low temperature is much richer than the usual two step melting process found previously in 2D Coulomb clusters and that temperature can induce also structural transitions which lead to an enhanced symmetry with increasing temperature.

In order to test if our results are still meaningful for other systems such as dusty plasmas and Coulomb balls we extended our investigation to systems with screened Coulomb interacting particles. The linear dynamics of the system is also investigated through a normal mode analysis.

The paper is organized as follows. In the next section our model system is introduced; the methodology used to find stable configurations and the normal modes are given. In Sec. III we deal with the system of classical particles interacting through a Coulomb interparticle potential. First, we investigate the melting processes in small clusters of particles ranging from $N=5$ to 13 particles, i.e., clusters with one shell. Subsequently we investigated larger systems and in particular the magic clusters with $N=19,32,38$, and 56 particles. In Sec. IV we investigate the melting processes which are relevant at very low temperature and in Sec. V systems of screened Coulomb interacting particles are addressed. Finally, in Sec. VI we present our conclusions.

\section{MODEL AND NUMERICAL APPROACH}

We study a 3D model system of $N$ equally charged particles in an isotropic confinement potential and interacting through a repulsive potential. The potential energy of the system is given by

$$
E=\sum_{i=1}^{N} \frac{1}{2} m \omega_{0}^{2} r_{i}^{2}+\sum_{i>j}^{N} V\left(\left|\mathbf{r}_{i}-\mathbf{r}_{j}\right|\right),
$$

where $m$ is the mass of the particle, $\mathbf{r}_{i}=\left(x_{i}, y_{i}, z_{i}\right)$ is the vector position of the $i$ th particle, $V(\mathbf{r})$ is the repulsive interparticle interaction potential, and $\omega_{0}$ is the confinement frequency of a single particle. In the present study we consider a screened Coulomb potential $V(\mathbf{r})=\left(q^{2} / \epsilon\right) \exp (-|\mathbf{r}| / \lambda) /|\mathbf{r}|$ which reduces to a Coulomb potential for $\lambda \rightarrow \infty$. We can write the potential energy (1) in dimensionless form

$$
E=\sum_{i=1}^{N} r_{i}^{2}+\sum_{j>i}^{N} \frac{\exp \left(-\kappa\left|\mathbf{r}_{i}-\mathbf{r}_{j}\right|\right)}{\left|\mathbf{r}_{i}-\mathbf{r}_{j}\right|},
$$

if we express the coordinates, energy, temperature, and time, respectively, in the following units $r_{0}=\left(q^{2} / \epsilon \gamma\right)^{1 / 3}$, where $\gamma$ $=m \omega_{0}^{2} / 2, E_{0}=\gamma r_{0}^{2}, T_{0}=E_{0} k_{B}^{-1}$, where $k_{B}$ is the Boltzmann constant and $t_{0}=\sqrt{2} / \omega_{0}$. The dimensionless inverse screening length, $\kappa=r_{0} / \lambda$, is a measure of the range of the interparticle interaction potential. All our numerical results will be given in dimensionless units.

The stable configuration is a local or global minimum of the potential energy which is only a function of the number of charged particles $N$ and the screened Coulomb parameter $\kappa$. Our numerical method to obtain the stable state configuration is based on the Monte Carlo simulation technique supplemented with the Newton method in order to increase the accuracy of the found energy value [16]. By starting from many different random initial configurations we are able to find the possible stable states, i.e., ground-state and metastable state configurations, which we documented in Ref. [14]. The eigenfrequencies are the square root of the eigenvalues of the dynamical matrix

$$
H_{\alpha \beta, i j}=\left.\frac{\partial^{2} H}{\partial r_{\alpha, i} \partial r_{\beta}, j}\right|_{r_{\alpha, i}=r_{\alpha, i}^{n}},
$$

where $\left\{r_{\alpha, i}^{n} ; \alpha=x, y, z ; i=1, \ldots, N\right\}$ is the position of the particles in the ground-state configuration.

\section{MELTING TRANSITION FOR SYSTEMS WITH COULOMB INTERPARTICLE POTENTIAL}

In this section we investigate the characteristics of the melting process in small 3D clusters of isotropically confined particles interacting via a Coulombic potential. In Ref. [19] magic clusters were investigated on the basis of potential energy calculations of the cluster configuration. This was done by calculating the addition energy, which was defined as

$$
\Delta(N)=[E(N+1)+E(N-1)-2 E(N)]
$$

where $E(N)$ is the ground-state energy of an $N$-particle cluster. Mathematically the addition energy corresponds to the second derivative of the potential energy $E(N)$ with respect to the total number of particles $N$, and as such describes the "curvature" of the energy as a function of the number of particles. Physically the addition energy is the amount of energy gained by the formation of two $N$-particle clusters out of two $(N-1)$ - and $(N+1)$-particle clusters. From the analysis of the minimum energy and the addition energy of the cluster Ref. [19] found that clusters with $N=6,12,19,32$, 38 , and 56 particles have large mechanical stability. Reference [20] also identified magic cluster configurations of 3D clusters. They implemented a topological analysis of the relative arrangement of particles in different shells and computed the Voronoi parameter. Furthermore the magic cluster configurations found in both references coincided for small 3D Wigner clusters. However, Refs. $[19,20]$ do not test directly the stability of $3 \mathrm{D}$ clusters. In this section we perform molecular dynamics (MD) simulation to investigate the mechanical stability of 3D isotropic Wigner crystals of charged particles interacting through a Coulomb interparticle interaction potential. From this investigation we were able to identify the correct magic clusters, i.e., those with pronounced mechanical stability.

The main static characteristics of the investigated 3D magic clusters are compiled in Table I. From left to right, Table I lists the number of particles in the system, its energy per particle, its configuration, the radius of the shells, and the width of the shells. The ground-state (GS) configuration of the systems with less than 13 particles form only one shell, while systems larger than 12 particles form two shells. For example, the third column of Table I shows that the system with $N=31$ has 4 and 27 particles, respectively, in the internal and external shells. From the last column of Table I, we 
TABLE I. From left to right: the number of particles in the system $(N)$, its energy per particle $(E / N)$, its configuration (conf.), the radius of the shell $(r)$, and the width of the shell $\Delta(r)$.

\begin{tabular}{|c|c|c|c|c|}
\hline$N$ & $E / N$ & Conf. & $r$ & $\Delta(r)$ \\
\hline 6 & 2.6540390 & 6 & 0.9406 & 0.0000 \\
\hline 12 & 4.8389665 & 12 & 1.2700 & 0.0000 \\
\hline \multirow[t]{2}{*}{18} & 6.6788303 & 1,17 & 0.0000 & \\
\hline & & & 1.5353 & 0.0012 \\
\hline \multirow[t]{2}{*}{19} & 6.9641459 & 1,18 & 0.0000 & \\
\hline & & & 1.5654 & 0.0122 \\
\hline \multirow[t]{2}{*}{20} & 7.2471808 & 1,19 & 0.0002 & \\
\hline & & & 1.5946 & 0.0176 \\
\hline \multirow[t]{2}{*}{31} & 10.0795110 & 4,27 & 0.7926 & 0.0124 \\
\hline & & & 1.9399 & 0.1098 \\
\hline \multirow[t]{2}{*}{32} & 10.3186788 & 4,28 & 0.7935 & 0.0000 \\
\hline & & & 1.9596 & 0.0882 \\
\hline \multirow[t]{2}{*}{33} & 10.5565871 & 4,29 & 0.7914 & 0.0215 \\
\hline & & & 1.9791 & 0.1304 \\
\hline \multirow[t]{2}{*}{37} & 11.4787472 & 6,31 & 0.9585 & 0.0324 \\
\hline & & & 2.0947 & 0.1150 \\
\hline \multirow[t]{2}{*}{38} & 11.7029516 & 6,32 & 0.9549 & 0.0000 \\
\hline & & & 2.1119 & 0.1166 \\
\hline \multirow[t]{2}{*}{39} & 11.9283228 & 6,33 & 0.9549 & 0.0348 \\
\hline & & & 2.1289 & 0.1186 \\
\hline \multirow[t]{2}{*}{55} & 15.2847026 & 12,43 & 1.2773 & 0.0250 \\
\hline & & & 2.4618 & 0.1086 \\
\hline \multirow[t]{2}{*}{56} & 15.4821444 & 12,44 & 1.2770 & 0.0193 \\
\hline & & & 2.4743 & 0.1110 \\
\hline \multirow[t]{2}{*}{57} & 15.6793502 & 12,45 & 1.2763 & 0.0210 \\
\hline & & & 2.4869 & 0.1110 \\
\hline
\end{tabular}

notice that the width of the external shell is larger than the width of the internal shell. In fact the external shell is formed by subshells each with a radius very close to each other. On the another hand, particles in small systems organize themselves in a perfect shell with width equal to zero, as we can see for the systems with $N=6$ and 12 particles. The stability of those subshells are investigated in Sec. IV.

In previous work on 2D systems [21] the melting temperature of the intrashell, intershell, and radial melting processes were determined. To do so the Lindemann criterion was used and the averaged displacements were computed. For a 3D system the expression for the square of the radial displacement becomes

$$
\Delta r_{\gamma}=\frac{1}{N_{\gamma}} \sum_{i=1}^{N_{\gamma}}\left\langle r_{i}^{2}\right\rangle-\left\langle r_{i}\right\rangle^{2}
$$

where $r_{i}$ is the modulus of the position vector of the $i$ th particle. Similarly, for the intrashell displacement we defined

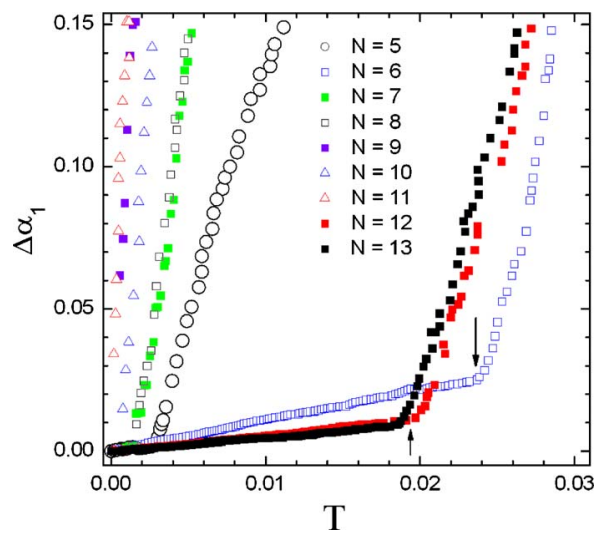

FIG. 1. (Color online) Intrashell displacement for systems ranging from $N=5$ to 13 particles as a function of the temperature.

$$
\Delta \alpha_{\gamma}=\frac{1}{N_{\gamma}} \sum_{i=1}^{N_{\gamma}}\left\langle\alpha_{i j}^{2}\right\rangle-\left\langle\alpha_{i j}\right\rangle^{2}
$$

where $\alpha_{i j}$ is the angle made by the position vectors of the two first neighboring particles of numbers $i$ and $j$ belonging to the same shell and

$$
\Delta \alpha_{\gamma \beta}=\frac{1}{N_{\gamma}} \sum_{i=1}^{N_{\gamma}}\left\langle\alpha_{i j}^{2}\right\rangle-\left\langle\alpha_{i j}\right\rangle^{2},
$$

for the intershell displacement, where $\alpha_{i j}$ is the angle made by the position vectors of the two first neighboring particles of numbers $i$ and $j$ belonging, respectively, to the $\beta$ th and $\gamma$ th shells. In both equations $N_{\gamma}$ indicates the total number of particles in the $\gamma$ th shell, and $\langle\cdots\rangle$ is an average over time. In order to characterize the melting temperature, we made use of a Lindemann-like criterion, which states that, close to the melting process, the respective averaged displacement starts to deviate rapidly from its low temperature linear dependence.

The dynamics of one-shell systems was investigated by a MD simulation where we collected data during an interval of $10^{6}$ time steps. The intrashell and radial displacements were computed. Figure 1 displays the results obtained for the intrashell displacement as a function of temperature for systems varying from $N=5$ to 13 particles. The intrashell melting temperatures of the magic clusters with $N=12$ and 6 particles are, respectively, equal to $T=0.0195$ and 0.0238 (indicated by black arrows in Fig. 1). Those critical temperatures are remarkably larger than the melting temperature found in other nonmagic clusters. The intrashell melting temperatures of magic clusters with $N=12$ and 6 particles are, respectively, about 7 and 8 times larger than the melting temperature $T=0.0027$ found for the cluster with $N=5$ particles, which is the nonmagic cluster of one shell structure that is most stable against intrashell diffusion. In fact, for increasing temperatures, the intrashell averaged displacement of nonmagic clusters of a single shell increases steeply once temperature becomes different from zero. The intrashell melting temperature for systems with $N=12$ and 13 particles have approximately the same value, i.e., $T=0.0195$ and 


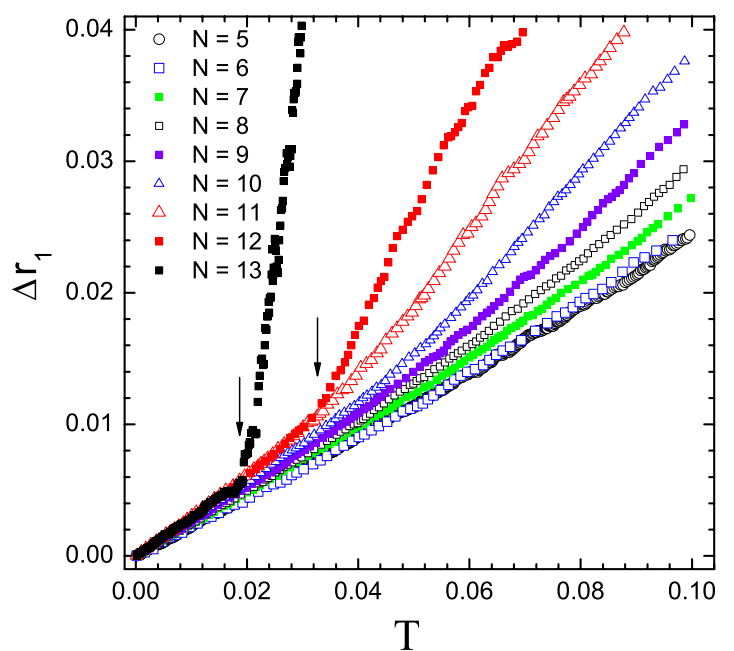

FIG. 2. (Color online) Radial displacement for systems ranging from $N=5$ to 13 particles as a function of the temperature.

0.0187 , respectively. This fact is not a surprise since both systems share the same arrangement of particles on the shell, i.e., particles arrange themselves in an icosahedral structure. One concludes that the GS configuration of the system with $N=13$ particles also forms a magic cluster. The latter fact could not be revealed through the addition energy analysis performed in previous works $[19,20]$.

Figure 2 displays the radial displacement against temperature for the ground-state configurations of systems ranging from $N=5$ to 13 particles. The largest system, i.e., the one with $N=13$ particles, is formed by one shell with 12 particles which encloses an extra particle sitting in the center. We see that the radial averaged displacement of this system changes its initial linear behavior to a rapid increase at a well defined critical temperature of $T=0.0194$. From previous experience obtained on 2D systems [22] we know that this rapid increase of the radial displacement occurs when the number of jumps of particles from the shell to the cluster's center and vice versa becomes statistically large. This is only possible due to the fact that the system with 13 particles has a metastable (MS) state with configuration $(1,12)$, i.e., one particle in the center and 12 particles forming a shell. Systems without metastable configurations are expected to expand gradually with increasing temperature. The curves for $\Delta r$ for systems with $N=5,6,7$, and 8 particles, which do not have metastable states, reflect correctly this statement, i.e., there is no abrupt change in their value. Finally we notice that for increasing temperatures the radial displacements of the clusters $N=9,10,11$, and 12 , which, respectively, have metastable configurations $(1,8),(1,9),(1,10)$, and $(1,11)$, also deviate from their low-temperature linear behavior. The larger the system the lower the radial melting temperature is. This can be clearly seen from the cases of $N=12$ and 13 particles whose radial melting temperatures are, respectively, equal to $T=0.0326$ and 0.0194 (indicated by black arrows in Fig. 2).

At very low temperature the dynamics of the system is ruled by simple harmonic oscillations about a local energy minimum. In this limit the melting process can also be understood through a normal mode analysis. Each mode is de-

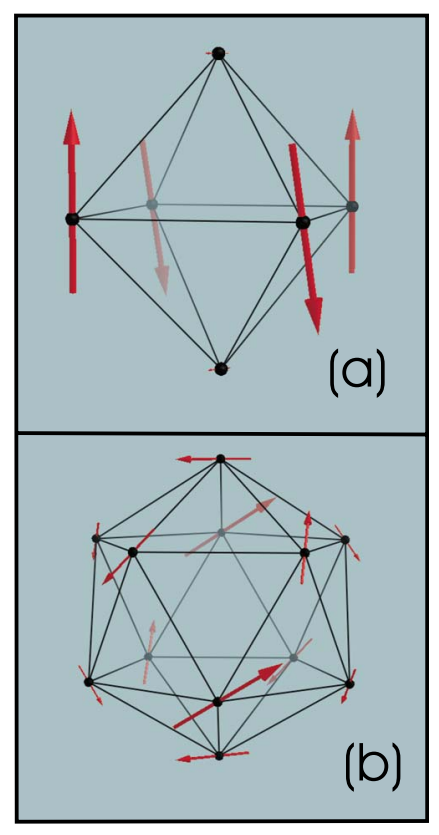

FIG. 3. (Color online) Representation (red arrows) of the oscillation mode of the first nonzero frequency for the systems with (a) $N=6$ and (b) 12 particles.

fined by an eigenvector and its corresponding eigenfrequency. Figures 3(a) and 3(b) represent the eigenvectors (red arrows) for the normal mode of the lowest nonzero frequency, respectively, for the systems with $N=6$ and 12 particles. The length of the arrows is proportional to the oscillation amplitude of the associated particle. The lowest frequency modes correspond to delocalized motions, in which a large number of particles oscillate with considerable amplitude. The highest frequency motions are more localized and contribute less to the melting process at low temperature. The first nonzero frequency should then be related with the first melting process that occurs in isotropically Wigner crystals if this melting process occurs at sufficiently low temperature. In other words, if the system has a large melting temperature, it will also have a large first nonzero frequency. The lowest nonzero frequency corresponds in fact to the fourth mode of oscillation since isotropic clusters always have three modes of rotation which frequencies are equal to zero [14]. Figure 4 displays the critical temperature of the first melting process (left axis) and the values for the first nonzero frequencies (right axis). We notice that the clusters with largest melting temperatures have also a large value for the first nonzero frequency. In particular the magic clusters with $N=6$ and 12 particles have, respectively, the frequencies $\omega=0.8516$ and 0.6654 , which are the two largest ones.

For fewer than 12 particles in a 3D system the groundstate configuration consists of a single shell. Those configurations in fact are three-dimensional regular polygons centered around the origin. For larger systems particle clouds arrange themselves in the form of concentric spherical shells if the confinement potential is isotropic. Those structures are classified by the number of particles per shell and they evolve as follows: for $N=13$ particles it becomes energetically favorable to have a single particle inside the shell; for 


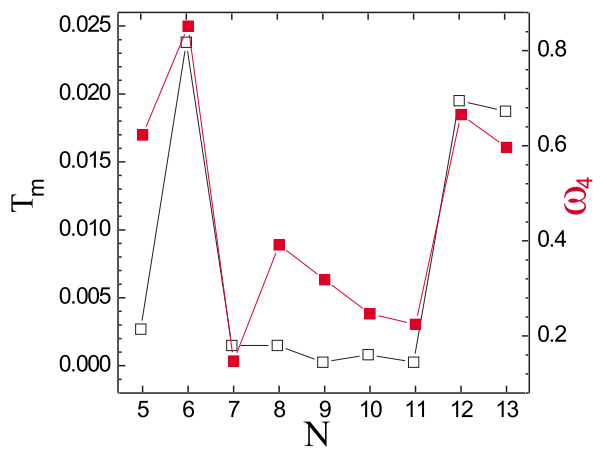

FIG. 4. (Color online) Critical temperature for the first melting process (left axis, black open square) and lowest nonzero frequency (right axis, red square) for systems varying from $N=5$ to 13 particles.

$22<N<27$ two particles constitute the internal structure, etc.; 12 is the maximum number of particles allowed to fill the internal shell which happens for the system with $N=60$ particles in the case of a two shell structure. In the case of ground state configurations consisting of two shells, the innershell structures are analogous in shape to those for the corresponding single shell clusters [23]. The latter statement strongly suggests that the knowledge about the dynamics of single shell systems is helpful in the understanding of the dynamics of larger systems.

Now we turn our attention to the investigation of the dynamics of larger systems, i.e., systems with two shells. The main goal of this investigation is trying to elucidate if magic number configurations present commensurability between particles in the same shell or/and in different shells. Such property was found to play an important role into the dynamics of small 2D clusters [15]. Analogously this commensurability in 3D systems should enhance the stability of the cluster leading to an enhanced melting temperature. To do so we compare the results of the intrashell, intershell, and radial displacements computed for the $\mathrm{N}$-particle magic cluster with the ones obtained for systems with $N-1$ and $N+1$ particles. Figure 5 displays the radial (black data), intrashell (blue data), and intershell (red data) deviations for the magic clusters with $N=32,38$, and 56 particles and for their respective neighbors. The radial and intrashell displacements were computed for the external shell [upper figures, i.e., Figs. 5(a), 5(c), and 5(e)] and internal shell [lower figures, i.e., Figs. 5(b), 5(d), and 5(f)].

If magic clusters have commensurability between particles belonging to different shells one should expect a large resistance against radial and/or intershell diffusion. Figure 5(a) displays the radial displacement (black symbols) computed for the particles in the external shell for systems with $N=31,32$, and 33 particles. Their radial melting temperatures are, respectively, equal to $T=0.0249,0.0206$, and 0.0172 [red arrows in Fig. 5(a)]. Those clusters follow the general behavior that the larger the cluster the lower the radial melting temperature. This is not hard to understand. It is built on the following two facts: (1) the number of metastable states increases with the number of confined particles and (2) metastable states contribute to a decrease of the melting temperature. Figure 5(e) displays the radial displacement (black symbols) for the external shell particles of the systems with $N=55,56$, and 57 particles. Their radial melting temperatures are, respectively, equal to $T=0.0154,0.0204$, and 0.0199 . The radial melting temperature of the magic cluster, i.e., the system with $N=56$ particles, is only slightly larger
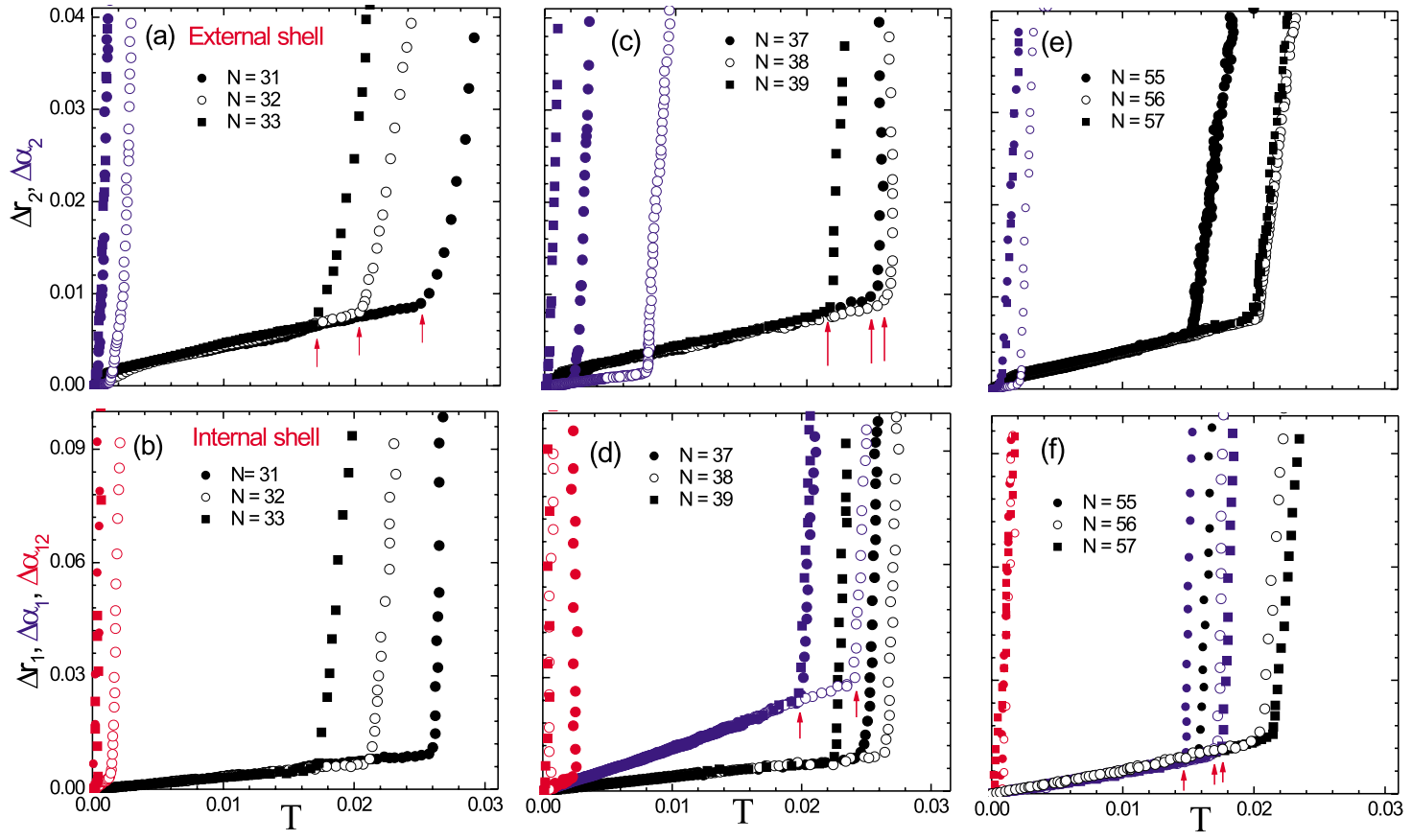

FIG. 5. (Color online) Radial (black color) and intrashell (blue color) displacement computed for the external (upper figures) and internal (lower figures) shells for clusters with a different number of particles. The red symbols in the lower figures give the intershell displacement for the different systems. 
than the one of the cluster with $N=57$ particles. The radial melting temperatures found for the external shell of the systems with $N=37,38$, and 39 particles are, respectively, equal to $T=0.0255,0.0265$, and 0.0221 and indicated by red arrows in Fig. 5(c). The magic cluster with $N=38$ particles has the largest radial melting temperature. We notice that large resistance against radial diffusion is not a unique property for magic clusters and that such a property is only found for the system with $N=38$ particles. We will see later that particles in the external shell of the system with $N=38$ particles organize themselves in a particular form. Finally we observe that for a given system the critical temperature of radial melting for the external shell [Figs. 5(a), 5(c), and 5(e)] and for the internal shell [Figs. 5(b), 5(d), and 5(f)] have approximately the same values. This fact is, of course, a consequence of the radial diffusion of particles close to the critical temperature. The effect of radial diffusion is expected to be strong in systems with multiple shells.

We have seen (Fig. 1) that 6 and 12 particles arrange themselves in a structure carrying special properties for their dynamics. This fact naturally led us to formulate the question: if those structures are surrounded by a second shell of particles, in the case of large systems, is their stability against melting retained? We found that the answer is positive, i.e., those structures keep their stability properties, as we will demonstrate now. First lets consider the case of six particles in the internal shell. Such situation occurs for the GS configuration of systems with structures keep their stability properties, as we will demonstrate now. First let us consider the case of six particles in the internal shell. Such a situation occurs for the GS configuration of systems with $N=37,38$, and 39 particles. The internal shell of the nonmagic clusters with $N=37$ and 39 particles have the same intrashell critical temperature of $T=0.0197$ while for the cluster with $N=38$ particles this critical temperature is $T$ $=0.0241[$ Fig. $5(\mathrm{~d})$, red arrows]. The value of those critical temperatures are lower than the intrashell melting temperature found for the isolated cluster with $N=6$ particles, i.e., $T=0.0238$ (Fig. 1). Also here we notice that the cluster with $N=38$ particles has the largest intrashell critical temperature. A similar picture is valid for the case when an icosahedron forms the internal shell of the systems with $N=55,56$, and 57 , where the intrashell melting temperatures in the internal shell are, respectively, equal to $T=0.0148,0.0174$, and 0.0177 [see Fig. 5(f), red arrows]. The lowering of the intrashell critical temperature of the internal shell of large clusters when compared to the ones of isolated clusters with $N$ $=6$ and 12 particles is easily understood. In larger clusters the icosahedral and octahedral structures are submitted to a fluctuating electric field generated by the thermal induced movement of the external shell particles.

The temperature dependence of the intrashell displacement computed for the external shell and the intershell displacement are shown, respectively, in Figs. 5(a) (blue symbols) and 5(b) (red symbols) for the systems with $N=31,32$, and 33 particles. We notice that for the same system those quantities diverge at the same temperature. For example, the intrashell displacement computed for the external shell [Fig. 5(a), blue open circles] and the intrashell displacement [Fig. 5 (b), red open circles] for the cluster with $N=32$ particles diverge both at the temperature of $T=0.00141$. Since those two quantities diverge at the same temperature we can only conclude that the intrashell melting on the external shell is the first melting process to occur and that the dynamics of those systems do not show any sign of intershell melting. An analogous situation is found for the nonmagic clusters $N$ $=31,33,37,39,55$, and 57. For those systems the intrashell displacement computed for the external shell and the intershell displacement also have the same critical temperature. This fact can be verified in Figs. 5(c) and 5(d) for the systems with $N=37$ and 39 particles and in Figs. 5(e) and 5(f) for the systems with $N=55$ and 57 particles.

The intershell melting plays an important role into the dynamics of the clusters with $N=38$ and 56 particles as we will now demonstrate. For the system with $N=38$ particles both the internal and external shells are relatively stable against intrashell melting, i.e., they are locked. The intrashell melting for the system with $N=38$ particles occurs at the large temperatures of $T=0.0241$ and $T=0.0078$, respectively, for the internal [Fig. 5(c)] and external [Fig. 5(d)] shells. In contrast to the large stability found against intrashell melting, this system exhibits low stability against intershell rotation. Figure 5(d) shows that its intershell displacement (red open circles) diverges immediately for increasing temperatures, i.e., at $T=0.0001$. When temperature is in the range $[0.0001$, $0.0084]$, i.e., the interval delimited by the critical temperatures, the dynamics is ruled by the relative motion of shells with respect to each other. The intershell rotation is also the first melting process to appear in the cluster with 56 particles. There the intershell and intrashell melting temperatures are, respectively, equal to $T=0.001$ and 0.002 . The range of temperature in which the intershell rotation is allowed is much larger for the magic cluster with $N=38$ particles than for the magic cluster with 56 particles.

We have found that the magic clusters with $N=6,12,13$, and 38 particles exhibit large resistance against intrashell diffusion. Furthermore the magic cluster with $N=38$ particles exhibits intershell melting. A common characteristic in the structure of those magic clusters is that they are composed of octahedral or icosahedral polygons.

The cluster with $N=19$ particles does not have one of those polygons, which makes its dynamics interesting as we will see further. Nevertheless, the system with 19 particles was classified in Refs. $[19,20]$ as a magic cluster. Figure 6 displays the radial (black symbols) and intrashell (blue symbols) displacements computed for the external shell of $N$ $=18,19$, and 20 particles. The radial melting temperatures for the clusters with $N=18,19$, and 20 particles are, respectively, equal to $T=0.051,0.040$, and 0.0315 and indicated in Fig. 6 (red arrows). The radial melting temperature follows the usual behavior, i.e., the larger the cluster the lower the melting temperature is. This system also does not present resistance against intrashell diffusion. A rapid increase of intrashell displacements (Fig. 6, blue symbols) for the systems with $N=18,19$, and 20 occurs immediately for increasing values of the temperature.

The first melting process for systems with one shell was found to occur at relatively high temperature if the first nonzero frequency is large. The first melting process for large clusters, i.e., clusters with two shells, is indicated by the red 


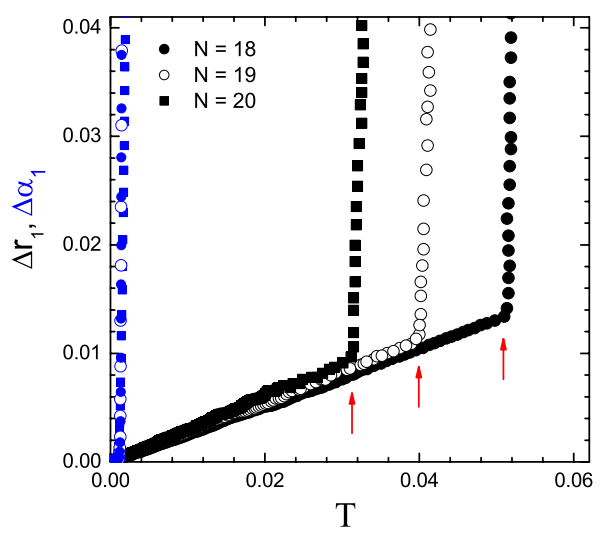

FIG. 6. (Color online) Radial (black color) and intrashell (blue color) displacements computed for the external shell of the systems with $N=18,19$, and 20 particles.

curve in Fig. 5. For the systems with $N=55,56$, and 57 particles the first melting process occurs almost immediately for increasing temperature while that for the clusters with $N=32$ and 37 particles have larger values for the critical temperature. Table II displays the value of the first nonzero frequency and the critical temperature of the first melting process. The systems with relatively large melting temperature and large first nonzero frequency are the ones with $N$ $=32,37$, and 56 particles. We notice that those systems are not necessarily the magic clusters. Our results obtained by MD simulations are in accordance with the cluster's stability estimated by the normal mode analysis both for clusters with one and two shells.

From the analysis of the dynamics of one shell systems, i.e., Figs. 1 and 2, it turns out that particles forming an octahedron and icosahedron have a large resistance against intrashell diffusion. We have seen that the system with $N=38$ particles has also high intrashell critical temperature for both shells. The stability found in the internal shell of the system with $N=38$ particles is due to its octahedral symmetry. The topological characteristic of the arrangement of particles on the surface of the shell can be characterized by their disclination charge $q$, which is the departure of their coordination number $c$ from the preferred coordination number $6(q=6$

TABLE II. From left to right: the number of particles in the system $(N)$, the temperature of the first melting process $\left(T_{m}\right)$, and the first lowest nonzero frequency $\omega_{4}$.

\begin{tabular}{lcc}
\hline \hline$N$ & $T_{m}$ & $\omega_{4}$ \\
\hline 31 & $3.12 \times 10^{-4}$ & 0.110 \\
32 & $1.37 \times 10^{-3}$ & 0.228 \\
33 & $3.12 \times 10^{-4}$ & 0.106 \\
37 & $2.30 \times 10^{-3}$ & 0.232 \\
38 & $4.99 \times 10^{-4}$ & 0.109 \\
39 & $2.05 \times 10^{-4}$ & 0.062 \\
55 & $2.36 \times 10^{-4}$ & 0.046 \\
56 & $8.16 \times 10^{-4}$ & 0.088 \\
57 & $6.33 \times 10^{-4}$ & 0.062 \\
\hline \hline
\end{tabular}

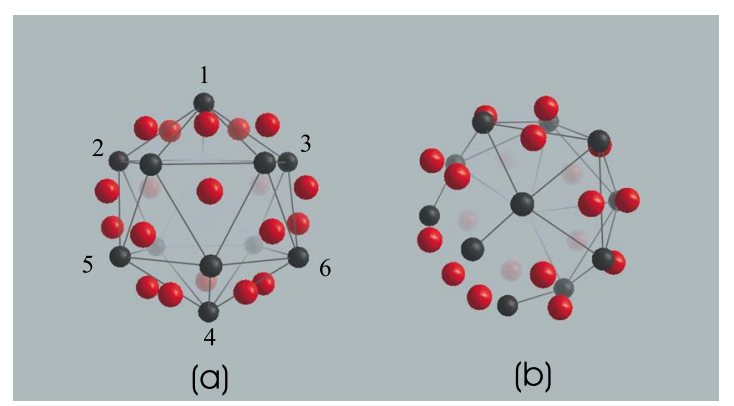

FIG. 7. (Color online) Particle arrangement of fivefold (black color) and sixfold (red color) particles on the outermost shell in the ground state of the system with $N=38$ (a) and 37 (b) particles. In order to improve visualization bounds between first neighboring fivefold particles are drawn.

$-c$ ). Particles with five and six neighbors are, respectively, called fivefold and sixfold particles. Figure 7(a) shows the arrangement of particles in the external shell for the system with $N=38$ particles. One notices that fivefold particles (in black color) form an icosahedral structure while the rest of the particles, i.e., sixfold particles (in blue color), arrange themselves around the corner of the icosahedron. Notice that in the external shell there is no situation where a pair of first neighboring particles is formed by two fivefold particles. In other words, there is at least one sixfold particle between any pair of fivefold particles. To improve visualization we display a projection of the top [Fig. 8(a)] and bottom [Fig. 8(b)] parts of Fig. 7. We notice that the relative arrangement of particles in Figs. 8(a) and 8(b) is equivalent and that the whole structures are rotated with respect to each other by an angle of $72^{\circ}$. Such an icosahedral structure formed by the fivefold particles in the external shell of the system with $N$ $=38$ particles is not found for other clusters. As one example we show the arrangement of particles in the external shell of the system with $N=37$ particles in Fig. 7(b). The structure formed by the fivefold particles is not an icosahedron and two fivefold particles can form a pair of first neighboring particles.

One of the most interesting results of this paper is the large resistance against intrashell diffusion found in the external shell of the GS configuration of the system with $N$ $=38$ particles. Next we investigated such mechanical prop-

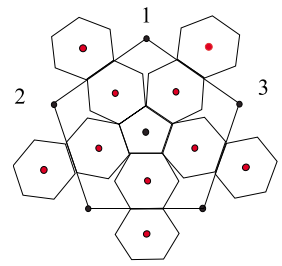

(a)

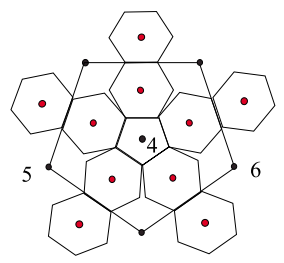

(b)
FIG. 8. (Color online) Visualization of the top (a) and bottom (b) of the arrangement of particles in the external shell of the GS configuration of the system with $N=38$ particles [Fig. 7(a)]. Particles in black and red are, respectively, fivefold and sixfold coordinated. 


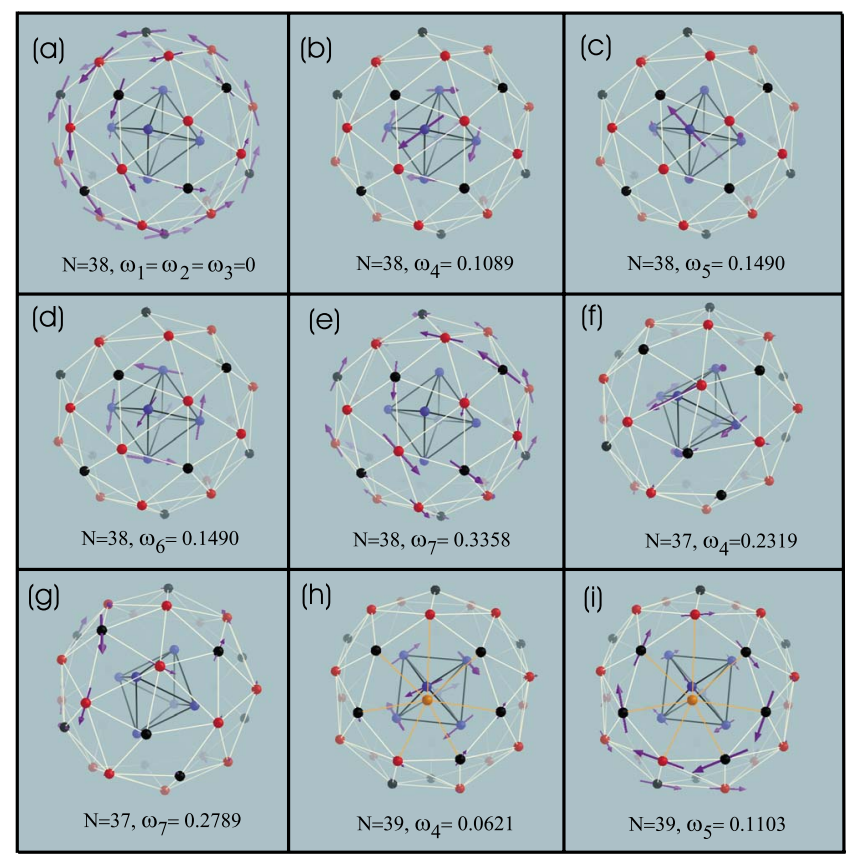

FIG. 9. (Color online) Visualization of the cluster structure and oscillation mode. For the external shell fivefold, sixfold, and sevenfold particles are represented, respectively, by black, red, and orange balls. Particles in the internal shell are represented by blue balls. The arrows represent the eigenvectors of a specific normal mode, and the arrow's length is proportional to the amplitude of oscillation of the associated particle.

erty through a normal mode analysis. To do so we first identify which oscillation modes correspond to diffusion of particles in the external shell. We took into account only the modes of lowest frequencies since delocalized modes are the most important to understand the melting process. 3D isotropic clusters have three degenerated modes of rotation with frequencies equal to zero. We show in Fig. 9(a) one of those modes for the GS configuration of the system with $N=38$ particles. In the external shell the fivefold and sixfold particles are indicated, respectively, by black and red balls. Particles in the internal shell are indicated by blue balls. The arrows indicate the direction of oscillation while their length is proportional to the amplitude of oscillation of each associated particle. Notice that for this mode particles in the internal and external shells oscillate approximately in parallel. In the sequence Figs. 9(b), 9(c), and 9(d) display, respectively, the fourth, fifth, and sixth oscillation modes, i.e., the first three lowest nonzero frequencies modes. Notice that those modes are governed mainly by the oscillation of particles in the internal shell characterizing an intershell rotation mode. Differently from the latter oscillation pattern, the seventh mode of the GS configuration of the system with $N$ $=38$ particles [Fig. 9(e)] exhibits an intrashell motion of the external shell. For this mode the amplitude of oscillation of particles is much larger in the external shell than in the internal one.

In order to characterize quantitatively those modes we define the averaged relative amplitude $\Delta v_{i}=v_{\text {ext }} / v_{\text {int }}$, where $v_{\text {ext }}\left(v_{\text {int }}\right)$ is the averaged amplitude of oscillation per particle

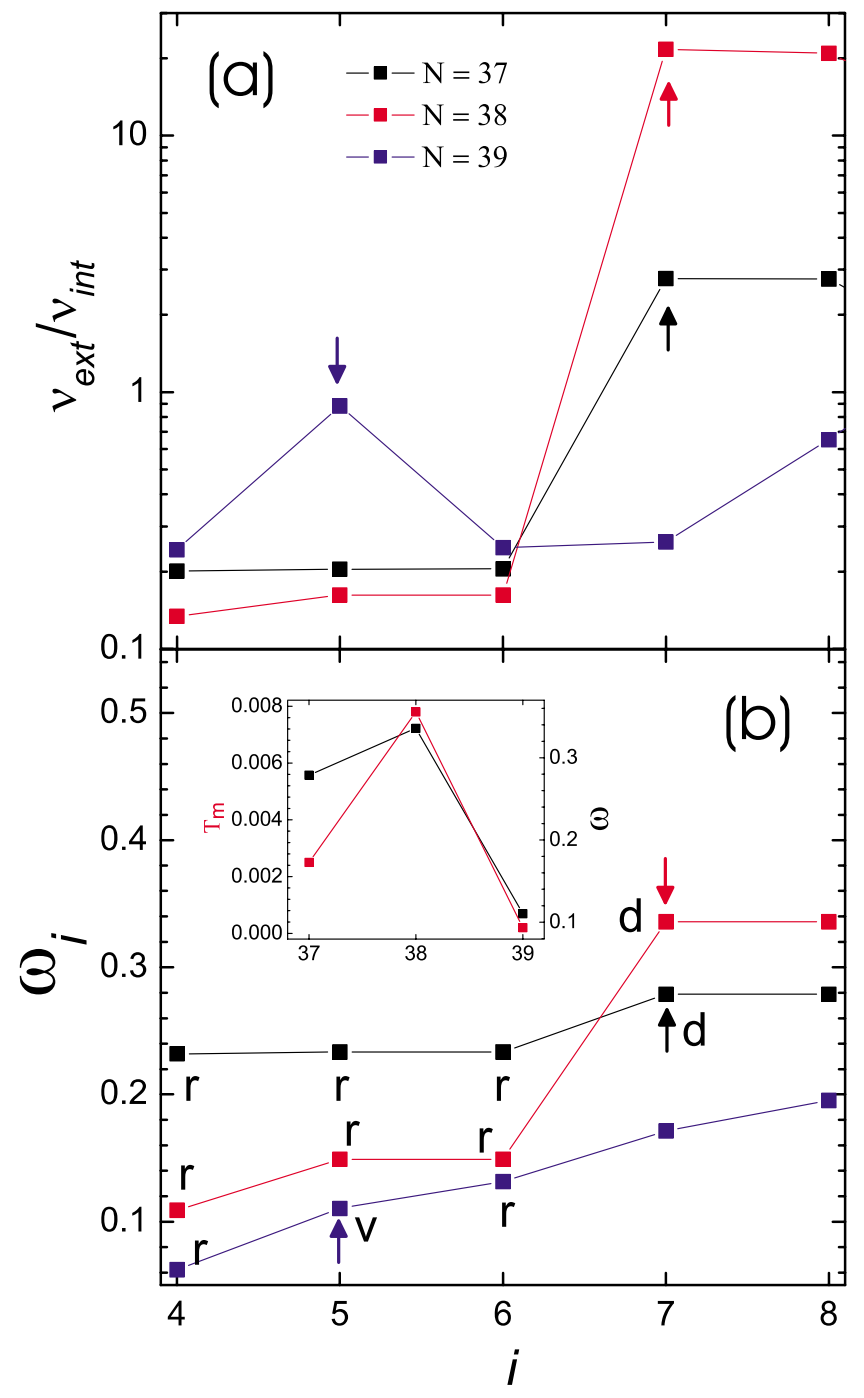

FIG. 10. (Color online) (a) Averaged relative amplitude and (b) eigenfrequencies for the systems with $N=37,38$, and 39 particles as a function of the mode number $i$ from $i=4$ to 8 . Arrows indicate the respective quantities related to intrashell melting. The letters state for $r$ (rotation), $v$ (vortex), and $d$ (diffusion of particles on the external shell). The inset displays the intrashell melting temperature (red squares, left axis) and the frequencies of the mode for intrashell motion (black open squares, right axis) for the systems with $N$ $=37$, 38, and 39 particles.

computed in the external (internal) shell for the $i$ th normal mode. The normal mode related to intrashell melting of the external shell is the lowest frequency mode with large value of $\Delta v_{i}$. Figure 10(a) displays $\Delta v_{i}$ as a function of the mode number $i$ for the systems with $N=37$ (black open squares), 38 (red squares), and 39 (blue squares) particles. In agreement with the analysis of Figs. 9(b), 9(c), and 9(d), the value of the averaged relative amplitude is very small for the first three lowest nonzero frequencies of the system with $N=38$ particles, i.e., $\Delta v_{i}<0.2$ for $i=4$, 5, and 6 [Fig. 10(a), red squares]. Contrarily, for the seventh mode of the system with $N=38$ particles the averaged relative amplitude is pronounced larger, i.e., $\Delta v_{7}=21.708$ [Fig. 10(a), indicated by red arrow]. The latter mode is related to the intrashell melt- 
ing of the external shell of the system with $N=38$ particles.

For the systems with $N=37$ and 39 particles the values of the averaged relative amplitude computed for the seventh and fifth modes are, respectively, equal to $\Delta v_{7}=2.7698$ [Fig. 10(a), black arrow] and $\Delta v_{5}=0.8852$ [Fig. 10(a), blue arrow]. Those are the modes with an enhanced value for the averaged relative amplitude and therefore must be related to the intrashell melting temperature on the external shell of those clusters. The eigenvector of the seventh oscillation mode of the system with $N=37$ particles is shown in Fig. $9(\mathrm{~g})$ while that of its rotation mode, i.e., the fourth one, is seen in Fig. 9(f). For the system with $N=39$ particles the fifth mode is shown in Fig. 9(i). This mode corresponds to a vortexlike motion where the vortex center sits at the sevenfold particle (orange ball). The mode of first nonzero frequency for the system with $N=39$ particles [Fig. 9(h)] corresponds mainly to an intershell rotation.

Figure 10(b) displays the eigenfrequencies for the systems with $N=37$ (black open squares), 38 (red squares), and 39 (blue circles) particles and mode number varying from $i=4$ to 8 . To indicate the motion mode the letters $r, v$, and $d$ are placed beside the data and stand, respectively, for rotation, vortex, and diffusion on the external shell. The frequencies of the modes characterized by intrashell motion are indicated by arrows. From Fig. 10(b) we can see that the rotation mode $\omega_{6}=0.1490$ for the system with $N=38$ particles is substantially smaller than its frequency for the intrashell mode, i.e., $\omega_{7}=0.3358$. The latter fact is in accordance with the values found for the critical temperatures of the system with $N$ $=38$ particles, i.e., an intershell melting temperature of value $T=0.0001$ [see Fig. 5(d), open red circles] which is much smaller than the intrashell critical temperature of the external shell $T=0.0078$ [see Fig. 5(c), open blue circles]. Furthermore the inset in Fig. 10(b) shows the values for the intrashell critical temperature of the external shell (left axis, red squares) and the frequency of the intrashell mode (right axis, black open squares) for the particle numbers $N=37,38$, and 39 . We notice a very good agreement between those two data, i.e., the larger the eigenfrequency, the higher the melting temperature is.

\section{FINE STRUCTURE}

Charged particles in 3D clusters, which are confined by a parabolic potential, order themselves in shells, if the number of particles is not too large. Often, such shells have a fine structure, i.e., the shells have a nonzero thickness (see last column of Table I) and closer inspection reveals that the shells consist of a few subshells, i.e., the shell has a nonzero width. In this section we consider the small temperature behavior of such clusters. We present a detailed investigation of the particle motion as a function of temperature before any jumps between shells occur and thus before the radial melting sets in. We found that the detailed behavior depends very crucially on the exact ground-state configuration and that temperature-induced structural transitions are possible which lead to an enhanced symmetry with increasing temperature.

Figure 11 shows the temperature dependence of the radial displacement computed for the external shell for the magic

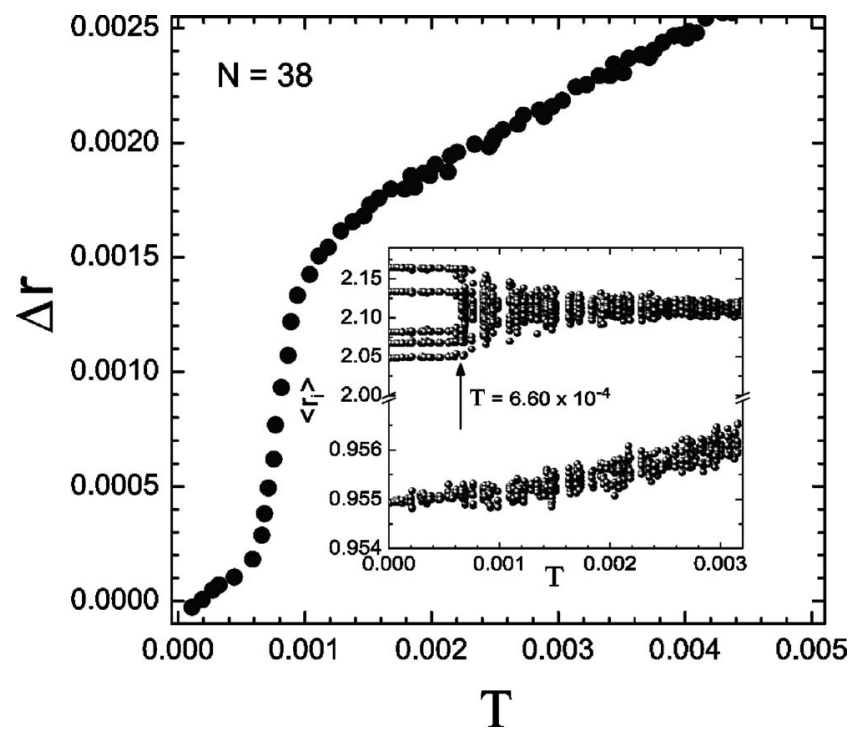

FIG. 11. Temperature dependence of the radial displacement for the system with $N=38$ particles in the low-temperature range. In the inset the average radial position of the particles is shown.

cluster with $N=38$ particles. We can see that radial displacement increases rapidly over a small temperature interval around $T=0.0014$. These small but rapid increases were never noticed before, probably because one needs rather accurate simulations in a small temperature range and for many temperature steps leading to long calculation times.

Such a phenomenon can be understood by investigating the average radial position of each particle as a function of temperature. These results are shown in the inset of Fig. 11, where at zero temperature the two-shell structure is clearly seen with the outer shell showing a fine structure resulting in five subshells. However, exactly at the temperature where the jump in the mean square radial deviation occurs for the outer shell, those subshells start to coalesce into a single shell. We can conclude that this jump corresponds to a local melting of the outer shells, forming a single broad shell. This leads to an increased symmetry of the system. Consequently, the transition is a temperature induced structural (phase) transition. It is similar to the structural phase transition which was recently found for certain 2D Coulomb bound clusters [24]. At this jump, the angular order between particles in different shells is destroyed, due to intershell motion, as shown in Figs. 5(c) and 5(d), which displays, respectively, the intrashell and intershell displacements.

The system with 38 particles is a magic configuration; it is interesting to see if the above results are different for a nonmagic configuration. We consider first the nonmagic cluster with $N=33$ particles. The radial displacement for the outer shell is shown in Fig. 12 and the average position of the particles in its inset. The outer (inner) shell can be divided into seven (two) subshells at zero temperature. With the slightest increase of temperature, the subshells start to form one broad shell. This local radial melting goes hand in hand with the loss of intrashell angular order [see Fig. 5(a)].

The radial displacement computed for the external shell of the system with $N=57$ particles is shown in Fig. 13 and in its 


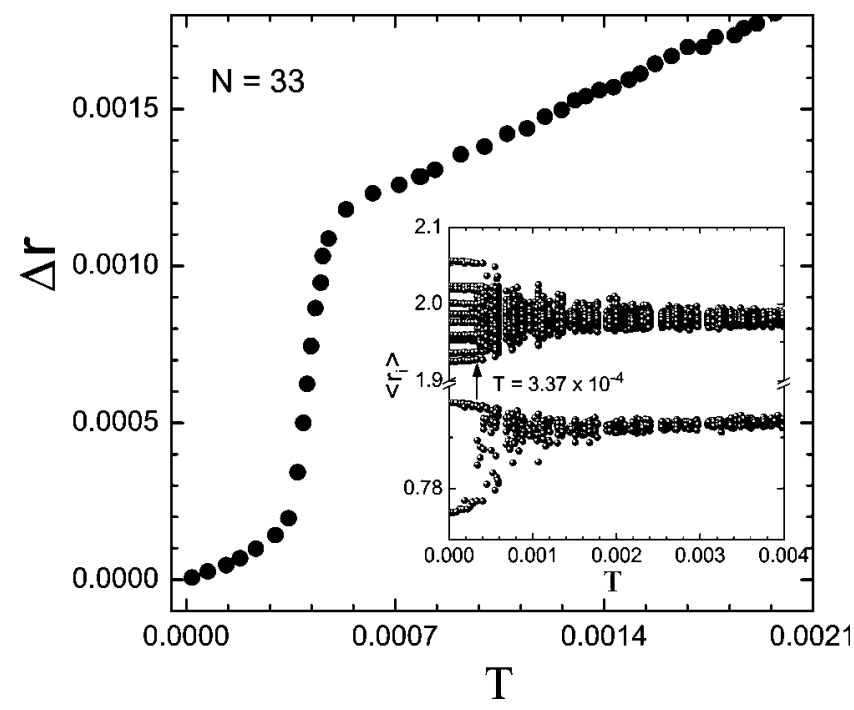

FIG. 12. The same as Fig. 11 but now for $N=33$ particles.

inset we can see the average radial position for all particles. The outer and inner shells at low temperature are composed of several subshells. For increasing temperature those subshells start to coalesce, but note that for the outer shell this is a two step process. Indeed, the radial displacement passes through two distinct jumps, respectively, at the temperatures $T=2.33 \times 10^{-4}$, where the outer shell merges into three subshells and $T=0.0012$ where finally the particles in the outer shell form a single broad shell. At those critical temperatures the pattern of the average radius changes, as we can see in the inset of Fig. 13. Furthermore, the orientational order between the first and second shells is already lost at the low critical temperature of $T=2.33 \times 10^{-4}$ [see Figs. 5(e) and $5(\mathrm{f})]$.

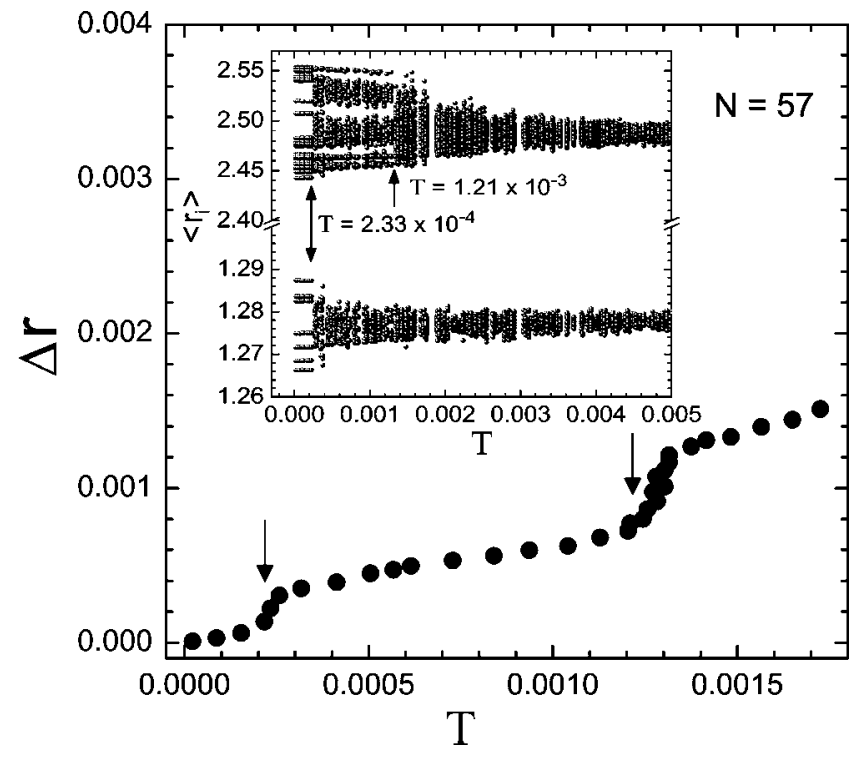

FIG. 13. The same as Fig. 11 but now for $N=57$ particles.

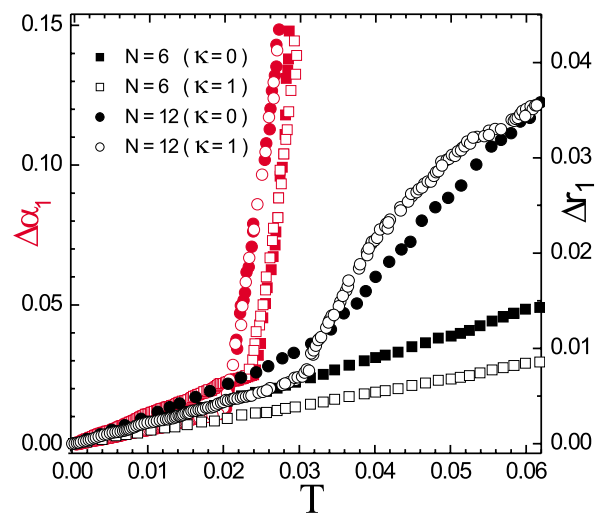

FIG. 14. (Color online) Temperature dependence of the intrashell (red symbols) and radial (black symbols) displacements for the magic clusters with $N=6$ and 12 particles and screening parameter $\kappa=1$ and 0 .

\section{MELTING TRANSITION FOR SYSTEMS WITH A SCREENED COULOMB INTERPARTICLE POTENTIAL}

In the previous sections we have investigated melting processes for isotropically confined Wigner crystals of particles interacting through a pure Coulomb potential. The dynamics of the systems was investigated over the whole temperature range. At high temperatures melting can develop through three processes, i.e., intrashell, intershell, and radial melting, while for larger clusters and at low temperatures a local transition sets in the external and internal shells which brings the system to a more symmetric configuration. However, many systems, for example, dusty plasmas, does not interact through a pure Coulomb potential but rather through a screened Coulomb interparticle potential. In this section we investigate the dependence of the melting processes on the range of the interparticle interaction. First, we investigate the melting process for magic clusters with $N=6$ and 12 particles and interacting through a screened Coulomb potential with screening parameter $\kappa=1$. This value of the screening parameter is typically found in experiments [25]. The characteristics of the melting process found for those systems will be compared with the one present in systems of particles interacting through a Coulomb potential. Finally, the effect of screening of the interparticle interaction potential in larger clusters, i.e., systems with $N=38$ and 39 particles, is addressed.

The temperature dependences of the intrashell displacement for the systems with $N=6$ and 12 particles and $\kappa=1$ are displayed in Fig. 14 (blue symbols). For the sake of comparison the results for the Coulomb case are also displayed. We note that there is no significant change in the behavior of the intrashell melting when the Coulomb potential is screened. The intrashell melting temperatures for the systems with $\kappa$ $=1$ and 0 have approximately the same values, i.e., $T$ $=0.0238$ and 0.0196 , respectively, for $N=6$ and 12 particles. The radius of the GS configuration decreases with increasing screening parameter. For the Coulomb $(\kappa=1$ screened Coulomb) case, the radius of the GS configurations are $r$ $=0.9406(0.8165)$ and $1.27(1.0646)$, respectively, for the 


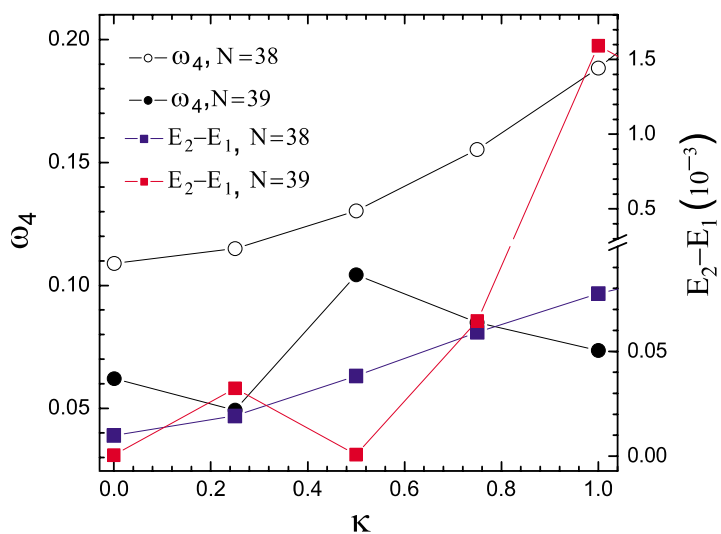

FIG. 15. (Color online) For the systems with $N=38$ and 39 particles: first nonzero frequency $\omega_{4}$ (left axis), and the energy difference $E_{2}-E_{1}$ between the lowest energy MS and the GS configuration (right axis) as a function of the screening parameter $\kappa$.

systems with $N=6$ and 12 particles, respectively. Although the radius of the cluster is reduced by increasing the value of $\kappa$ the relative angle between the position vectors of the particles in the GS configuration remains the same. In fact, particles in both GS configurations, i.e., for $\kappa=0$ and 1, arrange themselves in the corners of an icosahedron.

Figure 14 also displays the radial displacement against temperature for the GS configuration of the systems with $N$ $=6$ and 12 particles and screening parameter $\kappa=1$ (black data). The radial displacement for the Coulomb system was also added to the figure. We can see that the radial melting temperature also does not depend strongly on the screening parameter. The radial melting temperature for the system with $N=12$ particles is approximately $T=0.0326$ and 0.0332 , respectively, for $\kappa=0$ and 1 . Before the critical temperature we notice a slight difference in the linear behavior of the radial displacement. Actually, the radial displacement has a different slope for different values of $\kappa$ which was not the case for the intrashell displacement.

Now we analyze the effect of the screening of the interparticle interaction potential into the dynamics of large clusters. The screening parameter $\kappa$ can induce structural phase transitions of first and/or second order. At those transitions discontinuities in first and second derivatives of the energy with respect to the screening parameter are found [26,27]. This phenomenon can appear both in the GS and MS configurations and so the energy difference between those states is a good quantity to identify such structural phase transitions. Second order structural phase transitions can be identified through the computation of the first nonzero frequency. At the point where the second order transition occurs the first nonzero frequency becomes equal to zero [26]. Figure 15 displays the energy difference $E_{2}-E_{1}$ (right axis) between the lowest energy MS and the GS configurations against the screening parameter $\kappa$ for the systems with $N=38$ (blue squares) and $N=39$ (red squares) particles. We notice that the behavior of the energy difference strongly depends on the cluster size. The energy difference for the system with $N$ $=38$ particles increases smoothly with $\kappa$. The latter fact indicates that the screening parameter $\kappa$ does not induce a first

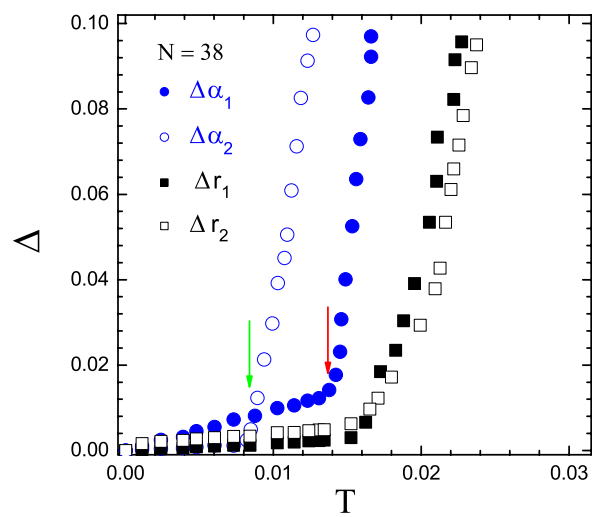

FIG. 16. (Color online) Temperature dependence of the intrashell and radial displacements computed for the internal and external shells of the magic cluster with $N=38$ particles and screening parameter $\kappa=1$.

order structural phase transition neither in the GS nor in the MS configurations of the system. In addition, Fig. 15 also displays the first nonzero frequency $\omega_{4}$ (left axis) for the system with $N=38$ (open circles) and $N=39$ (closed circles) particles. From the first nonzero frequency of the system with $N=38$ particles one can see that there is no second order structural phase transition since the first nonzero frequency does not go to zero. Actually, for a value of the screening parameter inside the interval $0 \leqslant \kappa \leqslant 1$ the GS and MS configurations of the clusters with $N=38$ particles keep the shell arrangement $(6,32)$. Differently, the energy difference $E_{2}$ $-E_{1}$ of the system with $N=39$ particles has many peaks which indicates that the GS or the MS configurations pass through a first order structural phase transition. In fact, we found that for its GS configuration the shell arrangements are $(6,33)$ and $(7,32)$, respectively, for $\kappa=0$ and $0<\kappa \leqslant 1$ while that for the MS configuration the shell arrangements are $(6,33),(7,32)$, and $(8,31)$, respectively, for $\kappa=0,0.25 \leqslant \kappa$ $\leqslant 0.75$ and $1 \leqslant \kappa \leqslant 2$. Such a structural phase transition might influence the melting behavior of the GS configuration. The latter will be discussed now for the systems with $N=38$ and 39 particles with $\kappa=1$.

Figure 16 displays the intrashell (blue data) and radial (black data) displacements for the internal and external shells of the systems with $N=38$ particles and screening parameter $\kappa=1$. The shell's occupation for the system with $N=38$ particles and $\kappa=1$ is $(6,32)$. The critical temperatures for the intrashell melting are $T=0.01378$ (Fig. 16, red arrow) and 0.0084 (Fig. 16, green arrow), respectively, for the internal and external shells. For the case of a pure Coulomb interparticle potential [Figs. 5(c) and 5(d)] the corresponding critical temperatures are $T=0.0241$ and 0.0078 . Those critical temperature are comparable in magnitude and it is clear that the screening of the interparticle interaction potential does not alter the main dynamical properties of the magic cluster with $N=38$ particles, i.e., a large intrashell melting temperature both in the internal and external shells.

Figure 17 displays the intrashell (blue data) and radial (black data) displacements for the internal and external shells of the systems with $N=39$ particles and screening parameter $\kappa=1$. The shell's arrangement for the system with $N=39$ par- 


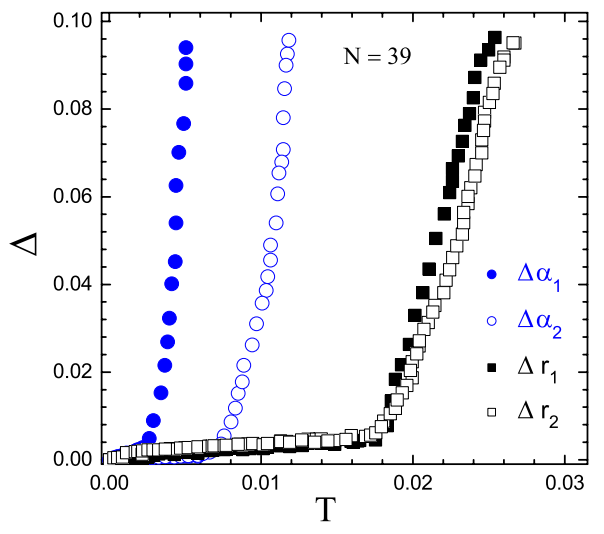

FIG. 17. (Color online) The same as Fig. 16 but now for $N$ $=39$ particles.

ticles and $\kappa=1$ is $(7,32)$. The intrashell melting temperature for the internal shell (Fig. 17, closed circles) is $T=0.0028$. This latter value is much lower than the one found for the case of a pure Coulomb interparticle potential, i.e., $T$ $=0.0197[$ Fig. 5(d)]. For the case of $\kappa=1$ the internal shell becomes less stable since seven particles do not make a magic arrangement. Oppositely, the stability of the external shell is enhanced and the intrashell melting temperature goes from $T=0.0003(\kappa=0)$ to $0.0069(\kappa=1)$. In fact, particles in the external shell of the system with $N=39$ particles and $\kappa$ $=1$ arrange themselves in a magic configuration similar to the one shown in Fig. 7(a).

\section{SUMMARY AND CONCLUSIONS}

We investigated the melting process of small 3D isotropically confined Wigner crystals of charged particles interacting through Coulomb or screened Coulomb potentials. The stability of the clusters was investigated by MD simulations where the Lindemann's criterion was used. We found that the GS configuration of the systems with $N=6,12,13$, and 38 particles have large mechanical stability and are therefore identified as the true magic clusters. The common characteristic of magic clusters is that they are formed by one of the highly regular structures, i.e., an octahedron or icosahedron. For the system with $N=38$ particles each fivefold coordinated particle sits in one of the corners of an icosahedron and is surrounded by sixfold coordinated particles.

We found that, due to the existence of MS configurations, the solid-liquid transition is rather sharp even in small size systems, i.e., systems with $N=12$ and 13 particles. Oppositely, the radial melting transition in smaller clusters, such as, for example, with $N=5$ to 8 particles, evolves continuously over a wide temperature interval. Systems with 6 and 12 particles have large intrashell melting temperature. Furthermore the resistance against intrashell diffusion in icosahedral and octahedral structures is found to remain large even when those structures form the inner shell of larger clusters.
We found that small size effects, i.e., the cluster's symmetry, plays an important role in the dynamics of small Wigner crystals. The melting transition can evolve through three different processes which strongly depend on the arrangement of particles on the shells of the GS configuration. The solid-liquid transition in nonmagic clusters occurs through two steps: first at low temperature the nonmagic cluster passes through an intrashell melting and then at higher temperature through a radial melting. Differently, pronounced resistance against intrashell diffusion is found in the magic cluster with $N=38$ particles which gives the system the possibility to undergo an intermediate melting process, the so-called intershell melting. This melting process was never studied before in 3D clusters.

The dynamics of systems with two shells where the external shell is formed by a set of subshells with slightly different radius was investigated in the small temperature range. It was shown that the radial order of those subshells in such a shell is maintained until the angular order between the different shells is lost. At this temperature, a jump in the mean square radial displacement is observed. This corresponds to a local melting of the shell resulting in larger radial fluctuations. This structural transition was not noticed before in $3 \mathrm{D}$ clusters. After this transition the symmetry of the cluster is increased and the particles in the broadened shell can interchange their positions. Such melting process is characteristic of the low-temperature dynamics and does not affect the stability of magic clusters.

The effect of screening of the interparticle interaction on the melting process was also investigated. The different critical melting temperatures for the systems with $N=6,12,38$, and 39 particles and $\kappa=1$ were determined. This value of the screening parameter is typical for experiments in dusty plasmas. We show that the effect of screening does not modify significantly the main characteristics of the dynamics found in the magic clusters as long as the configuration of the cluster is not modified. Furthermore, by changing the screening of the interparticle interaction potential one can induce structural phase transitions, i.e., where the configuration of the shell structure is modified, that enhances or decreases the stability of the shells of nonmagic clusters.

A normal mode analysis was performed and we found good agreement between the values of the eigenfrequencies and those of the critical temperatures obtained by MD simulation. From the normal mode analysis it turned out that (1) the oscillation mode associated to the first nonzero frequency has a strong character of intershell rotation in the case of a two shell configuration, (2) normal modes of the type intrashell motion has large eigenfrequencies if the system is a magic cluster, and (3) the fifth mode of the system with $N$ $=39$ particles presented a vortexlike motion around a sevenfold particle on its external shell.

\section{ACKNOWLEDGMENT}

This work was supported by the Flemish Science Foundation (FWO-Vl). 
[1] E. P. Wigner, Phys. Rev. 46, 1002 (1934).

[2] C. C. Grimes and G. Adams, Phys. Rev. Lett. 42, 795 (1979).

[3] E. Y. Andrei, G. Deville, D. C. Glattli, F. I. B. Williams, E. Paris, and B. Etienne, Phys. Rev. Lett. 60, 2765 (1988).

[4] J. H. Chu and Lin I, Phys. Rev. Lett. 72, 4009 (1994).

[5] Y. Kondo, J. S. Korhonen, M. Krusius, V. V. Dmitriev, E. V. Thuneberg, and G. E. Volovik, Phys. Rev. Lett. 68, 3331 (1992).

[6] W. M. Itano, J. J. Bollinger, J. N. Tan, B. Jelenković, X.-P. Huang, and D. J. Wineland, Science 279, 686 (1998).

[7] L. Hornekær, N. Kjærgaard, A. M. Thommesen, and M. Drewsen, Phys. Rev. Lett. 86, 1994 (2001).

[8] O. Arp, D. Block, A. Piel, and A. Melzer, Phys. Rev. Lett. 93, 165004 (2004).

[9] T. Pohl, T. Pattard, and J. M. Rost, Phys. Rev. Lett. 92, 155003 (2004).

[10] T. Killian, Nature (London) 429, 815 (2004).

[11] P. Buffat and J. P. Borel, Phys. Rev. A 13, 2287 (1976).

[12] J. P. Schiffer, Phys. Rev. Lett. 88, 205003 (2002).

[13] R. W. Hasse and V. V. Avilov, Phys. Rev. A 44, 4506 (1991).

[14] S. W. Apolinario, B. Partoens, and F. M. Peeters, New J. Phys. (to be published).

[15] V. M. Bedanov and F. M. Peeters, Phys. Rev. B 49, 2667 (1994).

[16] V. A. Schweigert and F. M. Peeters, Phys. Rev. B 51, 7700
(1995).

[17] G. Coupier, C. Guthmann, Y. Noat, and M. Saint Jean, Phys. Rev. E 71, 046105 (2005).

[18] B. G. A. Brito, J. N. T. Rabelo, and L. Cândido, Phys. Lett. A 353, 70 (2006).

[19] K. Tsuruta and S. Ichimaru, Phys. Rev. A 48, 1339 (1993).

[20] P. Ludwig, S. Kosse, and M. Bonitz, Phys. Rev. E 71, 046403 (2005).

[21] V. M. Bedanov, G. V. Gadiyak, and Y. E. Lozovik, Phys. Lett. 109A, 289 (1985)

[22] V. A. Schweigert and F. M. Peeters, J. Phys.: Condens. Matter 10, 2417 (1998).

[23] R. Rafac, J. P. Schiffer, J. S. Hangst, D. H. E. Dubin, and D. J. Wales, Proc. Natl. Acad. Sci. U.S.A. 88, 483 (1991).

[24] W. P. Ferreira, B. Partoens, F. M. Peeters, and G. A. Farias, Phys. Rev. E 71, 021501 (2005).

[25] M. Bonitz, D. Block, O. Arp, V. Golubnychiy, H. Baumgartner, P. Ludwig, A. Piel, and A. Filinov, Phys. Rev. Lett. 96, 075001 (2006)

[26] B. Partoens, V. A. Schweigert, and F. M. Peeters, Phys. Rev. Lett. 79, 3990 (1997); S. W. S. Apolinario, B. Partoens, and F. M. Peeters, Phys. Rev. E 72, 046122 (2005).

[27] L. Cândido, J. P. Rino, N. Studart, and F. M. Peeters, J. Phys.: Condens. Matter 10, 11627 (1998). 